PROCEEDINGS OF THE

AMERICAN MATHEMATICAL SOCIETY

Volume 136, Number 6, June 2008, Pages 2043-2053

S 0002-9939(08)09264-2

Article electronically published on February 21, 2008

\title{
A DECOMPOSITION THEOREM FOR FRAMES AND THE FEICHTINGER CONJECTURE
}

\author{
PETER G. CASAZZA, GITTA KUTYNIOK, DARRIN SPEEGLE, \\ AND JANET C. TREMAIN
}

(Communicated by N. Tomczak-Jaegermann)

\begin{abstract}
In this paper we study the Feichtinger Conjecture in frame theory, which was recently shown to be equivalent to the 1959 Kadison-Singer Problem in $C^{*}$-Algebras. We will show that every bounded Bessel sequence can be decomposed into two subsets each of which is an arbitrarily small perturbation of a sequence with a finite orthogonal decomposition. This construction is then used to answer two open problems concerning the Feichtinger Conjecture: 1. The Feichtinger Conjecture is equivalent to the conjecture that every unit norm Bessel sequence is a finite union of frame sequences. 2. Every unit norm Bessel sequence is a finite union of sets each of which is $\omega$-independent for $\ell_{2}$-sequences.
\end{abstract}

\section{INTRODUCTION}

The Kadison-Singer Problem [16] in $C^{*}$-Algebras has remained unsolved since 1959 , thereby defying the best efforts of several of the most talented mathematicians in our time. Recently, there has been a flurry of activity around this problem due to a fundamental paper by the first and fourth authors 11] (cf. also the longer version joint with M. Fickus and E. Weber [8]), which connects the Kadison-Singer Conjecture with many longstanding open conjectures in a variety of different research areas - in Hilbert space theory, Banach space theory, frame theory, harmonic analysis, time-frequency analysis, and even in engineering - by proving that these conjectures are in fact equivalent to the Kadison-Singer Problem.

In this paper we focus on the equivalent version of the Kadison-Singer Problem in frame theory, the so-called Feichtinger Conjecture. Before elaborating on the history of this conjecture and the contribution of our paper, let us first recall the basic definitions and notation in frame theory.

A countable collection of elements $\left\{f_{i}\right\}_{i \in I}$ is a frame for a separable Hilbert space $\mathcal{H}$, if there exist $0<A \leq B<\infty$ (the lower and upper frame bound) such that for

Received by the editors January 25, 2007.

2000 Mathematics Subject Classification. Primary 46C05, 42C15, 46L05.

Key words and phrases. Bessel sequence, decomposition, frame, Feichtinger Conjecture, frame sequence, Kadison-Singer Conjecture, $\omega$-independence, Riesz basic sequence.

The first author was supported by NSF Grant DMS 0405376.

The second author was supported by Deutsche Forschungsgemeinschaft (DFG) Research Fellowship KU 1446/5-1, by Preis der Justus-Liebig-Universität Gießen 2006, and by Deutsche Forschungsgemeinschaft (DFG) Heisenberg-Fellowship KU 1446/8-1.

The third author was supported by NSF Grant DMS 0354957.

(C) 2008 American Mathematical Society Reverts to public domain 28 years from publication 
all $g \in \mathcal{H}$,

$$
A\|g\|^{2} \leq \sum_{i \in I}\left|\left\langle g, f_{i}\right\rangle\right|^{2} \leq B\|g\|^{2} .
$$

A frame $\left\{f_{i}\right\}_{i \in I}$ is bounded, if $\inf _{i \in I}\left\|f_{i}\right\|>0$, and unit norm, if $\left\|f_{i}\right\|=1$, for all $i \in I$. Note that $\sup _{i \in I}\left\|f_{i}\right\|<\infty$ follows automatically from (1.1) by [5, Proposition 4.6]. If $\left\{f_{i}\right\}_{i \in I}$ is a frame only for its closed linear span, we call it a frame sequence. Those sequences which satisfy the upper inequality in (1.1) are called Bessel sequences. A family $\left\{f_{i}\right\}_{i \in I}$ is a Riesz basic sequence for $\mathcal{H}$, if it is a Riesz basis for its closed linear span, i.e., if there exist $0<A \leq B<\infty$ such that for all $\ell_{2}$-sequences of scalars $\left\{c_{i}\right\}_{i \in I}$,

$$
A \sum_{i \in I}\left|c_{i}\right|^{2} \leq\left\|\sum_{i \in I} c_{i} f_{i}\right\|^{2} \leq B \sum_{i \in I}\left|c_{i}\right|^{2} .
$$

Finally, a sequence $\left(f_{i}\right)_{i \in I}$ is called $\omega$-independent for $\ell_{2}$-sequences, if, whenever $c=\left(c_{i}\right)_{i \in I}$ is an $\ell^{2}$-sequence of scalars and $\sum_{i \in I} c_{i} f_{i}=0$, it follows that $c=0$.

Having recalled the necessary definitions and notation, we can now state the main conjecture we will be addressing in this paper.

Conjecture 1.1 (Feichtinger Conjecture). Every bounded frame can be written as a finite union of Riesz basic sequences.

Much work has been done on the Feichtinger Conjecture in just the last few years 15, 6, 12, 11, 8, 4. In particular, by employing the equivalence of the Paving Conjecture to the Kadison-Singer Problem shown by Anderson in 1979 [1] and by using the Bourgain-Tzafriri Conjecture [3] which arose from the "restricted invertibility principle" by Bourgain and Tzafriri from 1987 [2], the series of papers 6. 12, 11, 8 proves the equivalence between the Kadison-Singer Problem and the Feichtinger Conjecture. In [15] and [4] the Feichtinger Conjecture is considered for special frames such as wavelet and Gabor frames and frames of translates. For several classes of frames the Feichtinger Conjecture could indeed be verified, thereby verifing parts of the Kadison-Singer Problem.

Let us now take a closer look at the Feichtinger Conjecture (Conjecture 1.1). It is easily seen, by just normalizing the frame vectors, that we may assume in Conjecture 1.1 that the frame is a unit norm frame. It also follows easily that we only need to assume that the sequence is a bounded Bessel sequence. That is, by adding an orthonormal basis to the Bessel sequence we obtain a bounded frame which can be written as a finite union of Riesz basic sequences if and only if the original Bessel sequence can be written this way. Thus the Feichtinger Conjecture "reduces" to the conjecture that every unit norm Bessel sequence can be written as a finite union of Riesz basic sequences.

The first main result of our paper concerns a further reduction of the Feichtinger Conjecture. For this, consider the following conjecture which intuitively seems to be much weaker than Conjecture 1.1

Conjecture 1.2. Every unit norm Bessel sequence can be written as a finite union of frame sequences.

However, surprisingly, we will show that both conjectures are in fact equivalent.

Theorem 1.3. The Feichtinger Conjecture is equivalent to Conjecture 1.2. 
Part of the motivation of this result is a result by Casazza, Christensen, and Kalton [7] concerning frames of translates, which shows that the set of translates of a function in $L^{2}(\mathbb{R})$ with respect to an arbitrary subset of $\mathbb{N}$ is a frame sequence if and only if it is a Riesz basic sequence.

It is generally expected that the Kadison-Singer Problem will turn out to be false, which calls for positive partial results. Our second main result answers an open problem concerning weakenings of the famous Paving Conjecture of Anderson [1], which he showed is equivalent to KS. The main question has been whether every unit norm Bessel sequence is a finite union of $\omega$-independent sets. Recall that a set of vectors $\left\{f_{i}\right\}_{i=1}^{\infty}$ is $\omega$-independent if $\sum_{i=1}^{\infty} a_{i} f_{i}=0$ implies $a_{i}=0$ for all $i=1,2, \ldots$. This concept was defined in the 1950's by Marc Krein except that he requested that the above implication hold for sequences $\left\{a_{i}\right\}_{i \in I} \in \ell_{2}$. Since it is known [6] that every unit norm Bessel sequence is a finite union of linearly independent sets, our next main result gives that all unit norm Bessel sequences can be decomposed into a finite number of sets having Krein's $\omega$-independence.

Theorem 1.4. Every unit norm Bessel sequence which is finitely linearly independent is a union of two sets each of which is $\omega$-independent for $\ell_{2}$-sequences.

As a main ingredient for the proofs of Theorems 1.3 and 1.4, we will prove a decomposition theorem for frames, which is interesting in its own right. By providing an explicit construction, we will show that each unit norm Bessel sequence can be decomposed into two subsequences in such a way that both are small perturbations of "ideal" sequences. Our idea of an ideal sequence is a sequence for which there exists a partition of its elements into finite sets such that the spans of the elements of those sets are mutually orthogonal; thus, properties of the sequence are completely determined by properties of its local components. This definition is inspired by a more general notion called fusion frames [9, 10, which were designed to model distributed processing applications.

This paper is organized as follows. In Section 2 we will give the definition of $\epsilon$-perturbation, formalize the notion of an ideal sequence, and state some basic results. Section 3 contains the Decomposition Theorem and a discussion concerning an improvement of its proof and concerning the necessity of decomposing into two subsequences, whereas the proofs of Theorems 1.3 and 1.4 will be given in Section 4 .

\section{Definitions AND BASIC RESUlts}

For the remainder let $\mathcal{H}$ be a separable Hilbert space and let $I$ be a countable index set. Further, in the following we will write span $\left\{f_{i}\right\}_{i \in I}$ to mean the closed linear span of a set of vectors $\left\{f_{i}\right\}_{i \in I}$.

There exist many different definitions for a sequence being a perturbation of a given sequence. In this paper we will use the following.

Definition 2.1. Let $\left\{f_{i}\right\}_{i \in I}$ and $\left\{g_{i}\right\}_{i \in I}$ be sequences in $\mathcal{H}$ satisfying $\left\{g_{i}\right\}_{i \in I} \subset$ $\operatorname{span}_{i \in I}\left\{f_{i}\right\}$, and let $\epsilon>0$. If

$$
\sum_{i \in I}\left\|f_{i}-g_{i}\right\|^{2} \leq \epsilon
$$

then $\left\{g_{i}\right\}_{i \in I}$ is called an $\epsilon$-perturbation of $\left\{f_{i}\right\}_{i \in I}$.

For finite frames, it is precisely the interaction of the frame vectors which makes them interesting and applicable to a broad spectrum of applications. To employ 
the various results which were already obtained in this setting, an ideal infinite frame should allow its global properties to be determined locally by considering finite frame sequences. We formalize this idea in the next definition in the more general setting of an arbitrary sequence.

Definition 2.2. Let $\left\{f_{i}\right\}_{i \in I}$ be a sequence in $\mathcal{H}$. We say $\left\{f_{i}\right\}_{i \in I}$ possesses a finite orthogonal decomposition, if $I$ can be partitioned into finite sets $\left\{I_{j}\right\}_{j=1}^{\infty}$ so that

$$
\operatorname{span}_{i \in I}\left\{f_{i}\right\}=\left(\sum_{j=1}^{\infty} \oplus \operatorname{span}_{i \in I_{j}}\left\{f_{i}\right\}\right)_{\ell_{2}} .
$$

We wish to remark that the orthogonal family of finite dimensional subspaces forms an orthonormal basis of subspaces and in this sense is a special case of a Parseval fusion frame [9, 10.

The following lemma is well-known, but since the proof is short and it is fundamental to our construction, we include it for completeness.

Lemma 2.3. Let $\left\{f_{i}\right\}_{i \in I}$ be a Bessel sequence in $\mathcal{H}$, and let $P$ be a finite rank projection on $\mathcal{H}$. Then

$$
\sum_{i \in I}\left\|P f_{i}\right\|^{2}<\infty
$$

Proof. Let $\mathcal{K}$ be the projection space of $P$ with dimension $d$, and let $S$ denote the frame operator of $\left\{P f_{i}\right\}_{i \in I}$, i.e., $S(g)=\sum_{i \in I}\left\langle g, f_{i}\right\rangle f_{i}$ for all $g \in \mathcal{K}$. Further, let $\left\{e_{j}\right\}_{j=1}^{d}$ be an orthonormal eigenvector basis for $\mathcal{K}$ with respect to $S$ and respective eigenvalues $\left\{\lambda_{j}\right\}_{j=1}^{d}$. Then we obtain

$$
\sum_{i \in I}\left\|P f_{i}\right\|^{2}=\sum_{i \in I} \sum_{j=1}^{d}\left|\left\langle f_{i}, e_{j}\right\rangle\right|^{2}=\sum_{j=1}^{d} \sum_{i \in I}\left|\left\langle f_{i}, e_{j}\right\rangle\right|^{2}=\sum_{j=1}^{d} \lambda_{j}<\infty .
$$

\section{The Decomposition Theorem}

The following theorem states that we can decompose each unit norm Bessel sequence into two subsequences such that both are $\epsilon$-perturbations of sequences which possess a finite orthogonal decomposition. In fact, we will even derive an explicit algorithm for generating this partition. The proof is inspired by blocking arguments from Banach space theory [17. The Decomposition Theorem will also be the main ingredient for the proofs of Theorems 1.3 and 1.4 in Section 4.

Theorem 3.1 (Decomposition Theorem). Let $\left\{f_{i}\right\}_{i \in I}$ be a unit norm Bessel sequence in $\mathcal{H}$, and let $\epsilon>0$. Then there exists a partition $I=I_{1} \cup I_{2}$ such that, for $j=1,2$, the sequence $\left\{f_{i}\right\}_{i \in I_{j}}$ is an $\epsilon$-perturbation of some sequence in $\mathcal{H}$, which possesses a finite orthogonal decomposition.

Proof. Let $\left\{f_{i}\right\}_{i \in I}$ be a unit norm Bessel sequence in $\mathcal{H}$, and let $\epsilon>0$. Without loss of generality we may assume that $I=\mathbb{N}$, since if $I$ is finite we are done.

In the first step we will define a strictly increasing sequence $\left\{n_{i}\right\}_{i=1}^{\infty}$ in $\mathbb{N}$ by an induction argument. In the second step, we show that by defining $I_{j}:=$ $\bigcup_{i=0}^{\infty}\left\{n_{2 i+(j-1)}+1, \ldots, n_{2 i+j}\right\}$, for each $j=1,2$, the sequence $\left\{f_{i}\right\}_{i \in I_{j}}$ is an $\epsilon$ perturbation of some sequence $\left\{g_{i}\right\}_{i \in I_{j}}$ in $\mathcal{H}$, which possesses a finite orthogonal decomposition. 
For the initial induction step, we set $n_{1}:=1$. Further, we define $S_{1}$ by $S_{1}:=$ $\left\{n_{1}\right\}$, and let $P_{1}$ denote the orthogonal projection onto $\operatorname{span}_{i \in S_{1}}\left\{f_{i}\right\}$. To construct $n_{2}$, observe that, by Lemma 2.3 , we have

$$
\sum_{i=1}^{\infty}\left\|P_{1} f_{i}\right\|^{2}<\infty
$$

Therefore we can choose $n_{2}>n_{1}$ so that

$$
\sum_{i=n_{2}+1}^{\infty}\left\|P_{1} f_{i}\right\|^{2}<\frac{\epsilon}{2} .
$$

Using this new element of our sequence, we define $T_{1}$ by $T_{1}:=\left\{n_{1}+1, \ldots, n_{2}\right\}$ and let $Q_{1}$ denote the orthogonal projection onto $\operatorname{span}_{i \in T_{1}}\left\{f_{i}\right\}$.

We proceed by induction. Notice that in each induction step we will define two new elements of our sequence. Let $k \in \mathbb{N}$ and suppose that we have already constructed $n_{1}, \ldots, n_{2 k}$ and defined $\left\{S_{m}\right\}_{m=1}^{k},\left\{T_{m}\right\}_{m=1}^{k},\left\{P_{m}\right\}_{m=1}^{k}$, and $\left\{Q_{m}\right\}_{m=1}^{k}$. In the following induction step we will construct $n_{2 k+1}$ and $n_{2 k+2}$, and define $S_{k+1}$, $T_{k+1}, P_{k+1}$, and $Q_{k+1}$. First, we employ Lemma 2.3, which implies that

$$
\sum_{i=1}^{\infty}\left\|Q_{k} f_{i}\right\|^{2}<\infty
$$

Therefore we can choose $n_{2 k+1}>n_{2 k}$ so that

$$
\sum_{i=n_{2 k+1}+1}^{\infty}\left\|Q_{k} f_{i}\right\|^{2}<\frac{\epsilon}{2^{2 k}}
$$

Now let $S_{k+1}$ be defined by $S_{k+1}:=\left\{n_{2 k}+1, \ldots, n_{2 k+1}\right\}$, and let $P_{k+1}$ denote the orthogonal projection of $\mathcal{H}$ onto $\operatorname{span}_{i \in \cup_{m=1}^{k+1} S_{m}}\left\{f_{i}\right\}$. Secondly, again by Lemma 2.3. we have

$$
\sum_{i=1}^{\infty}\left\|P_{k+1} f_{i}\right\|^{2}<\infty
$$

Thus there exists $n_{2 k+2}>n_{2 k+1}$ such that

$$
\sum_{i=n_{2 k+2}+1}^{\infty}\left\|P_{k+1} f_{i}\right\|^{2}<\frac{\epsilon}{2^{2 k+1}} .
$$

Hence we define the set $T_{k+1}$ by $T_{k+1}:=\left\{n_{2 k+1}+1, \ldots, n_{2 k+2}\right\}$, and let $Q_{k+1}$ denote the orthogonal projection of $\mathcal{H}$ onto $\operatorname{span}_{i \in \cup_{m=1}^{k+1} T_{m}}\left\{f_{i}\right\}$. Iterating this procedure yields a sequence $\left\{n_{i}\right\}_{i=1}^{\infty}$ and, in particular, we obtain a partition $\left\{S_{m}\right\}_{m=1}^{\infty} \cup$ $\left\{T_{m}\right\}_{m=1}^{\infty}$ of $\mathbb{N}$.

For the second step let $\left\{S_{m}\right\}_{m=1}^{\infty}$ and $\left\{T_{m}\right\}_{m=1}^{\infty}$ be defined as in the induction argument. Then we define $I_{1}$ and $I_{2}$ by

$$
I_{1}:=\bigcup_{m=1}^{\infty} S_{m} \quad \text { and } \quad I_{2}:=\bigcup_{m=1}^{\infty} T_{m} .
$$

It remains to prove that, for $j=1,2$, we can construct a sequence $\left\{g_{i}\right\}_{i \in I_{j}}$ in $\mathcal{H}$ such that $\left\{g_{i}\right\}_{i \in I_{j}}$ has a finite orthogonal decomposition and $\left\{f_{i}\right\}_{i \in I_{j}}$ is an $\epsilon$-perturbation of it. 
In the following we will prove the claim only for $j=1$. The case $j=2$ can be dealt with in a similar manner. Using the sequence $\left\{P_{m}\right\}_{m=1}^{\infty}$ from the induction argument, we define $\left\{g_{i}\right\}_{i \in I_{1}}$ by

$$
g_{i}:=\left\{\begin{aligned}
f_{i} & : \quad i \in S_{1} \\
f_{i}-P_{m-1} f_{i} & : \quad i \in S_{m}, m>1 .
\end{aligned}\right.
$$

Since by construction we have

$$
g_{i} \in\left\{\begin{aligned}
P_{1} \mathcal{H} & : \quad i \in S_{1} \\
\left(P_{m}-P_{m-1}\right) \mathcal{H} & : \quad i \in S_{m}, m>1
\end{aligned}\right.
$$

hence

$$
\operatorname{span}_{1 \leq i<\infty}\left\{g_{i}\right\}=\left(\sum_{m=1}^{\infty} \oplus \operatorname{span}_{i \in S_{m}}\left\{g_{i}\right\}\right)_{\ell_{2}}
$$

it follows that $\left\{g_{i}\right\}_{i \in I_{1}}$ possesses a finite orthogonal decomposition. Further, for all $m \in \mathbb{N}$ and $i \in S_{m}$, we have

$$
P_{m-1} f_{i} \in \operatorname{span}_{k \in \bigcup_{l=1}^{m-1} S_{l}}\left\{f_{k}\right\}
$$

which implies that $\operatorname{span}_{i \in I_{1}}\left\{g_{i}\right\}=\operatorname{span}_{i \in I_{1}}\left\{f_{i}\right\}$. Finally, applying (3.1) and (3.3) yields

$$
\sum_{i \in I_{1}}\left\|g_{i}-f_{i}\right\|^{2}=\sum_{m=1}^{\infty} \sum_{i \in S_{m}}\left\|g_{i}-f_{i}\right\|^{2}=\sum_{m>1} \sum_{i \in S_{m}}\left\|P_{m-1} f_{i}\right\|^{2} \leq \sum_{m=2}^{\infty} \frac{\epsilon}{2^{m}}<\epsilon .
$$

Thus $\left\{f_{i}\right\}_{i \in I_{1}}$ is an $\epsilon$-perturbation of $\left\{g_{i}\right\}_{i \in I_{1}}$.

Remark 3.2. The decomposition argument can be done simultaneously on two frames at once - for example on a frame $\left\{f_{i}\right\}_{i \in I}$ and its dual frame, which is $\left\{S^{-1} f_{i}\right\}_{i \in I}, S$ being the frame operator of $\left\{f_{i}\right\}_{i \in I}$. We will not address this here, since we do not have any serious application at this time.

Next we observe that it is necessary to divide our index set into two subsets in Theorem 3.1. That is, the Bessel sequence itself need not be an $\epsilon$-perturbation of any sequence with a finite orthogonal decomposition.

Example 3.3. The unit norm Bessel sequence $\left\{f_{i}\right\}_{i=1}^{\infty}$ defined by $f_{i}=\frac{e_{i}+e_{i+1}}{\sqrt{2}}$ is not an $\epsilon$-perturbation of any sequence with a finite orthogonal decomposition for small $\epsilon>0$.

Proof. If we partition $\mathbb{N}$ into finite sets $\left\{I_{j}\right\}_{j=1}^{\infty}$, then there exists a natural number $i_{0} \in \mathbb{N}$ so that $i_{0} \in I_{j}$ and $i_{0}+1 \in I_{k}$ where $j \neq k$. Assume, by way of contradiction, that $\left\{g_{i}\right\}_{i=1}^{\infty}$ is an $\epsilon$-perturbation of $\left\{f_{i}\right\}_{i=1}^{\infty}$ and $\left\{g_{i}\right\}_{i=1}^{\infty}$ has a finite orthogonal decomposition given by $\left\{I_{j}\right\}_{j=1}^{\infty}$. Then $\left\|f_{i_{0}}\right\|=1$ and $\left\|f_{i_{0}}-g_{i_{0}}\right\|<\sqrt{\epsilon}$, which implies

$$
\left\|g_{i_{0}}\right\| \geq\left\|f_{i_{0}}\right\|-\left\|f_{i_{0}}-g_{i_{0}}\right\| \geq 1-\sqrt{\epsilon} .
$$

Similarly, $\left\|g_{i_{0}+1}\right\| \geq 1-\sqrt{\epsilon}$. Since $\operatorname{span}_{i \in I_{j}}\left\{g_{i}\right\}$ is orthogonal to $\operatorname{span}_{i \in I_{k}}\left\{g_{i}\right\}$, we have

$$
\left\|g_{i_{0}}-g_{i_{0}+1}\right\|^{2}=\left\|g_{i_{0}}\right\|^{2}+\left\|g_{i_{0}+1}\right\|^{2} \geq 2(1-\sqrt{\epsilon})^{2} \text {. }
$$


Using this estimate and the fact that $\left\|f_{i_{0}}-f_{i_{0}+1}\right\|^{2}=1$ and $\left\|f_{i_{0}}-g_{i_{0}}\right\|+\| f_{i_{0}+1}-$ $g_{i_{0}+1} \|<2 \sqrt{\epsilon}$, it follows that

$$
\begin{aligned}
\sqrt{2}(1-\sqrt{\epsilon}) & \leq\left\|g_{i_{0}}-g_{i_{0}+1}\right\| \\
& \leq\left\|f_{i_{0}}-f_{i_{0}+1}\right\|+\left\|f_{i_{0}}-g_{i_{0}}\right\|+\left\|f_{i_{0}+1}-g_{i_{0}+1}\right\| \\
& \leq 1+2 \sqrt{\epsilon} .
\end{aligned}
$$

This is a contradiction for small $\epsilon>0$.

Remark 3.4. Our proof of the Decomposition Theorem relies on the ordering of the elements of the sequence. This can sometimes cause problems, as we will see below. However, it is possible to do an optimal construction which removes this assumption. We first choose $i_{0} \in I$ and let $S_{1}=\left\{i_{0}\right\}$. Now, following the proof,

$$
\sum_{i \in I \backslash S_{1}}\left\|P_{1} f_{i}\right\|^{2}<\infty
$$

So choose $T_{1} \subset I \backslash S_{1}$ with $\left|T_{1}\right|$ minimal and

$$
\sum_{i \in I \backslash\left(T_{1} \cup S_{1}\right)}\left\|P_{1} f_{i}\right\|^{2}<\frac{\epsilon}{2}
$$

So we have put the $f_{i}, i \in I \backslash S_{1}$ with $\left\|P_{1} f_{i}\right\|$ maximal into $T_{1}$. In the induction step (equation (3.2)), choose

$$
S_{k+1} \subset I \backslash\left(\bigcup_{m=1}^{k} S_{m} \cup \bigcup_{m=1}^{k} T_{m}\right)
$$

with $\left|S_{k+1}\right|$ minimal and

$$
\sum_{i \in I \backslash\left(\bigcup_{m=1}^{k+1} S_{m} \cup \bigcup_{m=1}^{k} T_{m}\right)}\left\|Q_{k} f_{i}\right\|^{2}<\frac{\epsilon}{2^{2 k}} .
$$

Similarly, we now construct the next $T_{k+1}$ and then iterate the procedure.

This stronger form of the decomposition construction is useful because it eliminates the ordering of the elements. For example, if we work with the $\left\{f_{i}\right\}_{i=1}^{\infty}$ in Example 3.3. then for any permutation of $\left\{f_{i}\right\}_{i=1}^{\infty}$, as long as $f_{i_{0}}=f_{1}$, the decomposition we obtain from this stronger form of the proof of the Decomposition Theorem is

$$
\left\{\frac{e_{2 i-1}+e_{2 i}}{\sqrt{2}}\right\}_{i=1}^{\infty} \text { and }\left\{\frac{e_{2 i}+e_{2 i+1}}{\sqrt{2}}\right\}_{i=1}^{\infty},
$$

both of which are orthonormal bases for their spans. But, if we reorder the sequence $\left\{f_{i}\right\}_{i=1}^{\infty}$ by taking $\left\{f_{i}\right\}_{i=2^{k}}^{2^{k+1}-1}$ into

$$
\left\{f_{2^{k}+1}, f_{2^{k}+2}, \ldots, f_{2^{k+1}-1}, f_{2^{k}}\right\} \text { for each } 0 \leq k<\infty,
$$

the proof of the Decomposition Theorem produces the partition

$$
I_{1}=\left\{2^{2 k}, 2^{2 k+1}, \ldots, 2^{2 k+1}-1: 0 \leq k<\infty\right\}
$$

and

$$
I_{2}=\left\{2^{2 k+1}, 2^{2 k+1}+1, \ldots, 2^{2 k+2}-1: 0 \leq k<\infty\right\} .
$$


Now, $\left\{f_{i}\right\}_{i \in I_{j}}$ is not even a frame sequence for $j=1,2$. To see this for $I_{1}$, we note that the sets

$$
J_{k}=\left\{2^{2 k}, 2^{2 k+1}, \ldots, 2^{2 k+1}-1\right\} \quad(0 \leq k<\infty)
$$

give a finite orthogonal decomposition of $\left\{f_{i}\right\}_{i \in I_{1}}$ into linearly independent sets. So if $\left\{f_{i}\right\}_{i \in I_{1}}$ would be a frame sequence, then, since $\left\{f_{i}\right\}_{i \in I_{1}}$ is $\omega$-independent if and only if for each $k \in \mathbb{N}$ the sequence $\left\{f_{i}\right\}_{i \in J_{k}}$ is linearly independent and by [5, Proposition 4.3], it would also be a Riesz basic sequence. Let

$$
a_{2^{2 k}+i}=\frac{(-1)^{i}}{\sqrt{2^{2 k}}}, \quad i=0,1, \ldots, 2^{2 k}-1 .
$$

Then,

$$
\sum_{i=0}^{2^{2 k}-1}\left|a_{i}\right|^{2}=1
$$

while

$$
\left\|\sum_{i=0}^{2^{2 k}-1} a_{2^{2 k}+i} f_{2^{2 k}+i}\right\|^{2}=\left\|\frac{1}{\sqrt{2^{2 k}}}\left(e_{1}-e_{2^{2 k+1}-1}\right)\right\|^{2}=\frac{1}{2^{2 k-1}} .
$$

This implies that $\left\{f_{i}\right\}_{i \in I_{1}}$ is not a Riesz basic sequence. Thus $\left\{f_{i}\right\}_{i \in I_{1}}$ is not a frame sequence.

\section{Proofs of Theorems 1.3 and 1.4}

First we will prove Theorem 1.4. For this, we require a small change in the construction of Theorem 3.1. which can also be regarded as a strengthening due to the stronger conditions that the decomposition has to satisfy. However, notice that this modification can only be made provided we have a finitely linearly independent sequence.

Remark 4.1. We will alter the construction in the proof of Theorem 3.1 for finitely linearly independent sequences in the following way:

In the $k^{t h}$-step, instead of choosing $n_{2 k+1} \geq n_{2 k}$ such that

$$
\sum_{i=n_{2 k+1}+1}^{\infty}\left\|Q_{k} f_{i}\right\|^{2}<\frac{\epsilon}{2^{2 k}}
$$

we choose $n_{2 k+1} \geq n_{2 k}$ so that

$$
\sum_{i=n_{2 k+1}+1}^{\infty}\left\|Q_{k} f_{i}\right\|^{2}<\frac{\epsilon}{2^{2 k}} \delta_{k}
$$

where $\delta_{k}$ denotes the lower Riesz basis bound of $\left\{f_{i}\right\}_{i=1}^{n_{2 k}}$. The choice of $n_{2 k+2} \geq$ $n_{2 k+1}$ in the same step by (3.3) will be adapted similarly.

This now enables us to prove Theorem 1.4 . 
Proof of Theorem 1.4, Let $\left\{f_{i}\right\}_{i \in I}$ be a unit norm Bessel sequence which is finitely linearly independent. Further, let $\left\{T_{m}\right\}_{m=1}^{\infty},\left\{Q_{k}\right\}_{k=1}^{\infty}$, and $\left\{n_{k}\right\}_{k=1}^{\infty}$ be chosen as in the proof of Theorem 3.1 modified by Remark 4.1. We assume that there exists an $\ell_{2}$-sequence of scalars $\left\{a_{i}\right\}_{i \in T_{m}, m=1}^{\infty}$ such that

$$
\sum_{m=1}^{\infty} \sum_{i \in T_{m}} a_{i} f_{i}=0
$$

Fix $k \in \mathbb{N}$ and define $g_{k}$ by

$$
g_{k}:=\sum_{m=1}^{k} \sum_{i \in T_{m}} a_{i} f_{i}=-\sum_{m=k+1}^{\infty} \sum_{i \in T_{m}} a_{i} f_{i} .
$$

Recalling that $Q_{k}$ denotes the orthogonal projection of $\mathcal{H}$ onto $\operatorname{span}_{i \in \cup_{m=1}^{k} T_{m}}\left\{f_{i}\right\}$, by the definition of $g_{k}$, we have $Q_{k} g_{k}=g_{k}$. Employing this fact, we compute

$$
\begin{aligned}
\left\|g_{k}\right\|^{2} & =-\sum_{m=k+1}^{\infty} \sum_{i \in T_{m}} a_{i}\left\langle g_{k}, f_{i}\right\rangle \\
& \leq\left(\sum_{m=k+1}^{\infty} \sum_{i \in T_{m}}\left|a_{i}\right|^{2}\right)^{1 / 2}\left(\sum_{m=k+1}^{\infty} \sum_{i \in T_{m}}\left|\left\langle g_{k}, f_{i}\right\rangle\right|^{2}\right)^{1 / 2} \\
& \leq\left(\sum_{m=k+1}^{\infty} \sum_{i \in T_{m}}\left|a_{i}\right|^{2}\right)^{1 / 2}\left\|g_{k}\right\|\left(\sum_{m=k+1}^{\infty} \sum_{i \in T_{m}}\left\|Q_{k} f_{i}\right\|^{2}\right)^{1 / 2} .
\end{aligned}
$$

This implies

$$
\left\|g_{k}\right\| \leq\left(\sum_{m=k+1}^{\infty} \sum_{i \in T_{m}}\left|a_{i}\right|^{2}\right)^{1 / 2}\left(\sum_{m=k+1}^{\infty} \sum_{i \in T_{m}}\left\|Q_{k} f_{i}\right\|^{2}\right)^{1 / 2} .
$$

Now let $\delta_{k}$ denote the lower Riesz basis bound of $\left\{f_{i}\right\}_{i=1}^{n_{2 k}}$. Employing (4.1) and Remark 4.1] we obtain

$$
\begin{aligned}
\delta_{k} \sum_{m=1}^{k} \sum_{i \in T_{m}}\left|a_{i}\right|^{2} & \leq\left\|g_{k}\right\|^{2} \\
& \leq\left(\sum_{m=k+1}^{\infty} \sum_{i \in T_{m}}\left|a_{i}\right|^{2}\right)\left(\sum_{m=k+1}^{\infty} \sum_{i \in T_{m}}\left\|Q_{k} f_{i}\right\|^{2}\right) \\
& \leq\left(\sum_{m=k+1}^{\infty} \sum_{i \in T_{m}}\left|a_{i}\right|^{2}\right) \frac{\epsilon}{2^{2 k}} \delta_{k} .
\end{aligned}
$$

Thus,

$$
\sum_{m=1}^{k} \sum_{i \in T_{m}}\left|a_{k}\right|^{2} \leq \frac{\epsilon}{2^{2 k}} \sum_{m=k+1}^{\infty} \sum_{i \in T_{m}}\left|a_{i}\right|^{2} .
$$

Since $\left\{a_{i}\right\}_{i \in T_{m}, m=1}^{\infty}$, the left-hand-side of our inequality converges to the $\ell_{2}$ norm of this sequence of scalars while the right-hand-side converges to zero. It follows that $a_{i}=0$ for all $i=1,2, \ldots$.

Theorem 1.4 will now serve as the main ingredient in the proof of Theorem 1.3 . 
Proof of Theorem 1.3. Obviously, Conjecture 1.1 implies Conjecture 1.2

To prove the converse implication suppose that Conjecture 1.2 holds. If $\left\{f_{i}\right\}_{i \in I}$ is a unit norm Bessel sequence, it is in particular a finite union of linearly independent sets [6. Therefore, by Theorem 1.4, it is a finite union of sets which are $\omega$-independent for $\ell_{2}$-sequences. If $\left\{f_{i}\right\}_{i \in J}$ is one of these families, by our assumption it is a finite union of frame sequences. But each of these frame sequences is $\omega$-independent for $\ell_{2}$-sequences, hence it is a Riesz basic sequence.

\section{ACKNOWLEDGMENTS}

The majority of the research for this paper was performed while the second and third authors were visiting the Department of Mathematics at the University of Missouri. These authors thank this department for its hospitality and support during their visit. We also thank the American Institute of Mathematics for hosting the workshop "The Kadison-Singer Problem" which gave us additional inspiration and also the possibility to work out the last details of the results presented in this paper.

\section{REFERENCES}

1. J. Anderson, Extensions, restrictions, and representations of states on $C^{*}$-algebras, Trans. Amer. Math. Soc. 249 (1979), 303-329. MR 525675 (80k:46069)

2. J. Bourgain and L. Tzafriri, Invertibility of "large" submatrices with applications to the geometry of Banach spaces and harmonic analysis, Israel J. Math. 57 (1987), 137-224. MR890420 (89a:46035)

3. J. Bourgain and L. Tzafriri, On a problem of Kadison and Singer, J. Reine Angew. Math. 420 (1991), 1-43. MR1124564 (92j:46104)

4. M. Bownik and D. Speegle, The Feichtinger conjecture for wavelet frames, Gabor frames and frames of translates, Canad. J. Math. 58 (2006), 1121-1143. MR2270922 (2007h:42044)

5. P. G. Casazza, The art of frame theory, Taiwanese J. of Math. 4 (2000), 129-201. MR1757401 (2001f:42046)

6. P. G. Casazza, O. Christensen, A. Lindner, and R. Vershynin, Frames and the Feichtinger conjecture, Proc. Amer. Math. Soc. 133 (2005), 1025-1033. MR2117203 (2006a:46024)

7. P. G. Casazza, O. Christensen, and N. Kalton, Frames of translates, Collect. Math. 52 (2001), 35-54. MR1833085 (2002f:42002)

8. P. G. Casazza, M. Fickus, J. C. Tremain, and E. Weber, The Kadison-Singer problem in mathematics and engineering: a detailed account, in: Operator Theory, Operator Algebras, and Applications, D. Han, P. Jorgensen, and D. R. Larson, eds., Contemp. Math. 414, Amer. Math. Soc., Providence, RI (2006), 299-356. MR2277219 (2007j:42016)

9. P. G. Casazza and G. Kutyniok, Frames of subspaces, in: Wavelets, Frames, and Operator Theory, C. Heil, P. E. T. Jorgensen, and D. R. Larson, eds., Contemp. Math. 345, Amer. Math. Soc., Providence, RI (2004), 87-113. MR2066823 (2005e:42090)

10. P. G. Casazza, G. Kutyniok, and S. Li, Fusion Frames and Distributed Processing, Appl. Comput. Harmon. Anal. (to appear).

11. P. G. Casazza and J. C. Tremain, The Kadison-Singer problem in mathematics and engineering, Proc. Natl. Acad. Sci. USA 103 (2006), 2032-2039. MR2204073 (2006j:46074)

12. P. G. Casazza and R. Vershynin, Kadison-Singer meets Bourgain-Tzafriri, preprint (2005).

13. O. Christensen, An Introduction to Frames and Riesz Bases, Birkhäuser, Boston, 2003. MR.1946982 (2003k:42001)

14. O. Christensen and A. Lindner, Decomposition of Riesz frames and wavelets into a finite union of linearly independent sets, Lin. Alg. Appl. 355 (2002), 147-159. MR 1930142 (2003g:42057)

15. K. Gröchenig, Localized frames are finite unions of Riesz sequences, Adv. Comput. Math. 18 (2003), 149-157. MR 1968117 (2004a:42044) 
16. R. Kadison and I. Singer, Extensions of pure states, American Jour. Math. 81 (1959), 383-400. MR 0123922 (23:A1243)

17. J. Lindenstrauss and L. Tzafriri, Classical Banach Spaces I: Sequence Spaces, Springer-Verlag, Berlin, 1977. MR0500056 (58:17766)

Department of Mathematics, University of Missouri, Columbia, Missouri 65211

E-mail address: pete@math.missouri.edu

Program in Applied and Computational Mathematics, Princeton University, PrinceTON, New JeRSEy 08544

E-mail address: kutyniok@math.princeton.edu

Department of Mathematics and Computer Science, Saint Louis University, St. Louis, Missouri 63103

E-mail address: speegled@slu.edu

Department of Mathematics, University of Missouri, Columbia, Missouri 65211

E-mail address: janet@math.missouri.edu 